\title{
Medical doctors and community engagement in the aftermath of a disaster
}

\author{
Rosaria Indah*1,2 \\ ${ }^{1}$ Fakultas Kedokteran, Universitas Syiah Kuala, Banda Aceh, Indonesia \\ ${ }^{2}$ Pusat Riset Ilmu Sosial dan Budaya, Universitas Syiah Kuala, Indonesia
}

\begin{abstract}
Medical doctors conduct many health care and promotion activities after a disaster. However, there are limited studies on how doctors engage disaster-affected community members during the activities. This paper aims to examine medical doctors' experiences in engaging their community during the rehabilitation period after a big disaster. The study is based on a more extensive ethnographic study at disaster-affected health centers and communities in Aceh. The researcher conducted interviews with eleven community doctors and 45 hours of participant observations involving three doctors. Findings show different levels of community engagement in the doctor participants' experiences. Most of them only 'informed' rather than 'engaged' the disaster-affected patient and community. This paper calls for the enactment of community engagement in healthcare practices and education.
\end{abstract}

\section{Introduction}

Disasters may cause significant disruptions in healthcare services for several reasons: healthcare facilities may be severely damaged, deaths of healthcare workers. and patients might be displaced to areas afar. These disruptions provide significant challenges for medical doctors to cope. Therefore, medical doctors working in disaster areas should be prepared to face the challenges together with the community they serve. Medical doctors are not well-positioned to respond to disasters without proper training and link to public health officials and community elements [1]. Yet, there is scant research on how doctors engage disaster-affected communities in healthcare.

This paper aims to explore the life experiences of medical doctors working in post-disaster areas. It also examines how they engaged community members to cope with challenges in the aftermath of a huge disaster. It starts with a literature review on the medical doctor's role in disaster management, concepts of community engagement, and elaboration on data collection and data analysis methods. Subsequently, the findings will be explained and discussed further. The paper concludes and suggests some recommendations for further research.

\subsection{Medical doctor's role in disaster management}

Disaster may expose medical doctors to many uncertainties, both personally and in the community. These uncertainties hinder medical doctors from working synergically with community members to manage the disaster's effects [2].

Disaster management works in different phases connected in a cycle: Emergency responses, reconstruction, rehabilitation (post-disaster phases), and these efforts are designed to prevent, mitigate and prepare (pre-disaster stages) for the next possible disaster. Medical doctors play essential roles depending on the stages of disaster management, both before the disaster and its aftermath [3].

Within the stages of disaster management, doctor roles slightly transform from conventional roles [2]. Before a disaster, the ultimate task of medical doctors is to increase the population's disaster resilience by improving their health status. In the aftermath, doctors' primary mission is to rebuild the health status and reduce health disparity between the disaster-affected and nonaffected communities. To do so, doctors need to engage the targeted post-disaster community in close collaboration to assure health programs achieve an optimum result [4].

\subsection{Community engagement: concept, principles, and frameworks}

There are several concepts of community engagement in healthcare. The first one was inspired by Paul Farmer [5], who applied 'liberation theology' to address public health issues, such as tuberculosis (TB) in low-income and developing countries (LIDCs). Liberation theology argues that authentic transformation is rooted in marginalized communities.

\footnotetext{
*Corresponding author: rosariaindah@unsyiah.ac.id
} 
To activate the transformation, one needs to consider a simple methodology consisting of three linear steps: (i) observe, (ii) judge, and (iii) act[5]. Observing includes various elements, such as seeking the causes of the problem, followed by eliciting the experiences and views of a marginalized community and then incorporating the result of judgment and actions [5]. The second step, judgment, is produced by careful observation that should be directed to raise the consciousness of people and explain the processes of coming to an understanding of how social structures can cause injustice [5]. The act linear step is more than just reporting one's findings because it also requires accompanying a marginalized community in its struggle for liberation.

Applying the idea of liberation theology, Farmer's project in Haiti incorporated a program of funding food vouchers, offering travel expenses, and supplying Tuberculosis (TB) drugs for TB patients. Initially, Farmer observed that failures in previous TB treatments were due to a lack of community engagement [5]. When he asked community members why the last treatment of TB failed, they told him they could not afford to buy food or travel to health centers to collect their TB drugs. Based on this finding, it was concluded that food vouchers were to be incorporated because the root of the cause was malnutrition and structural poverty [5]. By increasing the participation and role of community members in TB treatment, Farmer had addressed the problem of managing TB cases effectively.

There are principles in community engagement in healthcare [6]. First, healthcare practitioners have to map existing networks and resources. If the healthcare program uses infrastructures, it is essential to make the community take ownership of the structures and maintain them. Third, cultural competence [4], [7], fourth, ensure two-way communication, and fifth, engage vulnerable groups, build trust and acknowledge existing culture.

Several organizations developed several frameworks of community engagement, such as Health Canada [8], US Clinical and Translational Science Award [9], and Association for Public Participation [10]. These three frameworks were quite similar, underlining gradual steps to increase the level of power-sharing (see Fig. 1.).

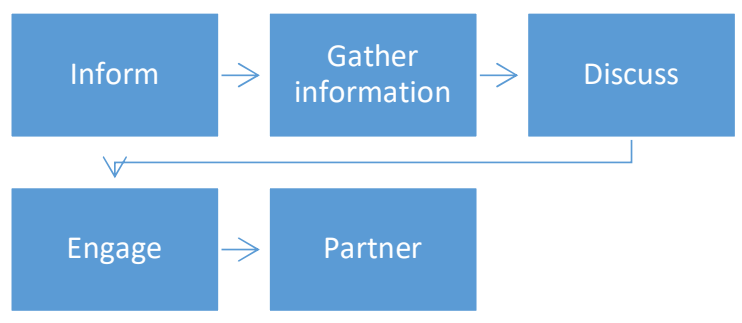

Fig. 1. Public involvement continuum, by Health Canada [8].

However, different terms were used. They described the lowest level of engagement as informing or educating, followed by listening, consulting, involving/engaging, and collaborating. The highest level of engagement was partnering with shared decision-making and leadership. A comparative study of the approaches concluded that there was a need to provide robust studies on the success of implementation and the extent of community engagement because current publications only explain these approaches at the theoretical level without adequate empirical data [11].

These models help provide a general understanding of different levels of engagements in community-based healthcare projects to align with the spirit of powersharing. The lower levels of engagement, such as 'consulting,' appreciate the situated knowledge possessed by the community. In comparison, the moderate to higher levels of engagement (involve, collaborate, and shared-leadership) seek to recognize the community's ability to speak and therefore act. However, one must be aware that the lowest level of engagement (educate or inform) potentially perpetuates the effects of asymmetrical power relations between healthcare providers and patients.

\section{Method}

This study was a part of a larger ethnographic research. It used ethnographic tools to explore medical doctors' experiences engaging disaster-affected patients and communities after the 2004 Indian Ocean tsunami in Aceh, Indonesia. The province of Aceh was the devastated province in Indonesia, in which most of its coastlines were hit by the 2004 Indian Ocean tsunami. The official death toll was 166,760 , while 635,384 survived the disaster but were internally displaced. Among the survivors, there were 1,736 people injured, and $61 \%$ of them lost one or more immediate family members that exposed them to a greater risk of mental illnesses such as depression, anxiety, and post-traumatic stress disorder (PTSD). Some of the participants also experienced the loss of core family members and assets.

The inquiry was set in natural settings, post-disaster healthcare facilities. Ethnographic tools allowed the researcher to explore cultural patterns and perspectives of medical doctors in natural environments. Although this methodology required a prolonged contact to locate interpretations about those occurrences into profound scripts [12], ethnographic tools may help depict the participants' meanings [13]. Ethnographic tools were also helpful to focus on social processes that facilitate daily life [14], especially on those related to community engagement. To collect data, the researcher conducted interviews and participant observations.

\subsection{Interview}

This data collection method aimed to understand medical doctors' perspectives upon their interactions with communities affected by the 2004 Indian Ocean tsunami in Aceh. The author chose to conduct interviews with experienced medical doctors working in post-tsunami areas, which have been there for at least ten years.

There were some challenges in conducting the interviews. First, it was difficult to locate medical 
doctors who had been working in the disaster-affected area for such an extended period (more than ten years). Most of the doctors working in the aftermath of the tsunami have moved to other places outside the affected areas after their two years of new graduate mandatory service had been completed. Only some of them decided to continue their works in those underserved areas. By conducting the snow-ball method, some medical doctors can be located. They were willing to spend some of their precious time for one or more interviews.

Second, when the researcher was trying to obtain their consent, some doctors were reluctant due to the time constraint. The researcher's identity as a medical doctor did not necessarily help her attain consent. They rejected because they assumed that the researcher could discern the circumstances of a high intensity of emotion and workload, with great responsibilities and stakes involved. Hence, the researcher decided to treat them as 'busy informants' [15] She waited until they offered her a time to meet.

Other challenges include locating and setting the interview. The researcher must travel more than $600 \mathrm{~km}$ from her main area to reach some participant doctors. However, it was worth spending time since most of them were willing to raise the issues that were central to her research topic.

Third, although most doctor participants agreed to be interviewed; however, one was reluctant to recall 'horrible memories' related to her personal experiences in the 2004 Indian Ocean tsunami that may trigger their PTSD. To prevent coerciveness, the researcher reminded the participant's right. She may decide to cease or continue with the interview at any point in time. The doctor participant, who was initially reluctant, chose to continue.

Other doctors have asked the researcher to wait and provide some time to deal with a potential emotional discomfort caused by remembering their experiences after the traumatic event. Talking about their experiences regarding disasters, death, and suffering is almost always categorized as a 'difficult conversation' in the medical world that needs careful mental preparedness [16]. The researcher expressed her understanding and patiently waited for their consent. She realized that gaining access to disaster-affected participants is one of the challenges that disasterfocused researchers encounter. However, most of the participants eventually agreed when they discussed the information statements and understood the risk and benefit associated with their involvement within the research.

Another way to obtain consent was by asking other medical colleagues to be a gatekeeper. They facilitated the interviews with targeted informants. An ethnographer should seek a gatekeeper's help in ethnographic research, such as what 'Doc' did in the classic ethnography piece 'Street Corner Society.' The gatekeeper provided a more accessible entry to the field. In this case, the participants admitted that they conducted 'a research' on the researcher's identity and credibility before granting their approval on setting up the interviews.

This 'research on the researcher's credibility is partly due to the assumption that the researcher might be obtaining financial gain from the research or might be a 'spy' investigating their 'good practice.' Fortunately, the researcher's experience as a teacher provided her with networking with former students. They acted as gatekeepers and thus verified researcher's identity and credibility. Eventually, she succeeded in obtaining consent from most of her planned participants. Yet, some of them have refused to be audio-recorded.

Individual semi-structured interviews were conducted with 11 community doctor participants. In the beginning, the researcher explained the aim of this study and information written on the participant information sheet. Participants may ask any questions regarding the research being answered.

Most interviews lasted 45 to 90 minutes. The interviews were flexible, interactive, dialogic, and reflective. An interview guide was used to support and organize the responses conveyed in Bahasa Indonesia. All doctors chose their office as the location for their interview. The characteristics of doctor participants are described in Table 1.

Table 1. Doctor participants characteristics.

\begin{tabular}{|c|l|c|c|c|}
\hline No & \multicolumn{1}{|c|}{ Font } & Sex & Age & $\begin{array}{c}\text { Experience } \\
\text { (years) }\end{array}$ \\
\hline 1 & Dr. Alia & F & 40 & 10 \\
\hline 2 & Dr. Jalal & M & 45 & 12 \\
\hline 3 & Dr. Endang & F & 40 & 17 \\
\hline 4 & Dr. Aisha & F & 55 & 20 \\
\hline 5 & Dr. Fajar & M & 54 & 25 \\
\hline 6 & Dr. Eva & F & 55 & 20 \\
\hline 7 & Dr. Julie & F & 54 & 21 \\
\hline 8 & $\begin{array}{l}\text { Dr. } \\
\text { Mahmud }\end{array}$ & M & 55 & 12 \\
\hline 9 & Dr. Anna & F & 50 & 15 \\
\hline 10 & Dr. Sarah & F & 53 & 12 \\
\hline 11 & Dr. Rita & F & 54 & 20 \\
\hline
\end{tabular}

\subsection{Participant observation}

To further understand how the doctor participants engaged their community in healthcare, the researcher chose three out of 11 community medical doctors interviewed. Before the participant observation was conducted, the objectives were explained in detail, and consent was sought since the observation was a more intrusive method of data collection. During the observation process, sometimes the researcher attended clinical encounters as a full observer and sometimes helped them as their administrative assistant or aid.

Initially, the researcher felt uncertain about how to conduct the observation, how to dress or participate in the different settings around health centers in villages. The researcher was unsure about what to expect because 
the setting was unfamiliar to her. Therefore, the researcher allowed doctors to define the boundaries within clinical encounters. The researcher asked them about her attire, where she should sit, when she should join in a conversation, ask or answer questions, or whether she should not. The researcher tried not to observe merely. She listened actively to their doctorpatient conversations to capture any means of engagement processes. The community doctors introduced the researcher to their patients and informed them that she was there to observe doctor-patient interactions. The doctors or the researcher asked for each patient's consent to be observed. If any patient refused permission, the researcher exited the examination room. For example, one male patient who did not want to be observed asked the researcher to stop her observation. The community medical doctor participants allowed the researcher to participate with community members inside and outside the health center, such as in health promotion campaigns, home visits, and non-clinical events, such as luncheons and wedding ceremonies of family members, or just enjoying coffee with community chiefs. As a result, rich descriptions of data were produced from these observations. The data from participant observations were documented in a logbook, field notes, and research memos.

\subsection{Data analysis}

Data analysis included carefully reading and rereading the transcripts of interviews, field notes, writing research memos, and generating codes. Some codes were generated deductively from the literature on the first round of data reading. Others were inductively produced from the data corpus at the second and third rounds of reading the entire data. Then the researcher made a coding scheme and categorized data into relevant categories based on these codes to generate preliminary themes. The author then did the fourth round of data reading before conducting the next round of thematic analysis to finalize research themes. The data analysis process was facilitated by NVivo version 10 software and guided by the study's research question, which included life experiences of medical doctors in engaging their communities in the aftermath of the 2004 Indian Ocean tsunami. In the writing up of the analysis, the names of all medical doctors were given pseudonyms to protect their anonymity.

\section{Findings}

As mentioned previously, the 2004 Indian Ocean tsunami devastated, destroyed, and damaged houses and displaced survivors from their homes [17]. Survivors were forced to flee and find new dwellings, often recovering from injury. Challenges related to health issues and internal displacement were intertwined, creating a complicated situation that needed to be understood by community medical doctors working in the aftermath of a disaster. Hence, patients in this context required special attention.
One day the researcher was allowed to observe the practice of Dr. Mahmud, a camp doctor, for a week. He had been working in a subdistrict with a high prevalence of tuberculosis, one of the effects of the overcrowded displacement situation. He used local languages, such as Achehnese or Aneuk Jamee, to converse with patients accordingly. He explained the reason:

Language could be a big problem for doctors. I want to engage patients in conversation about therapy. Therefore, they need to understand the details of the treatment offered. Unfortunately, I see most new doctors do not have the skill to speak the local languages, maybe because they came from different cultural backgrounds. (Dr. Mahmud, male, 55 years old)

It seems that Dr. Mahmud had successfully engaged his patients and their family members in the course of their treatment. During the observed conversations, Dr. Mahmud asked questions, responded to patients' questions, used jokes to establish a good rapport. He also made sure patients understood their essential roles in the treatment processes. However, Dr. Mahmud had never mentioned or suggested patients utilize resources in their communities, such as nutrition and sanitation programs in their community health centers. He engaged his patients at individual and family levels.

Other medical doctors expanded their engagement at the community level. To improve access to healthcare provisions for disaster-affected patients, community medical doctors often conducted outreach programs, as explained by Dr. Rita.

I went to mosques or people's houses to provide healthcare services and health promotions, mostly in religious community gatherings where people were gathered, even when they happened outside working hours. Even though I was not paid to do home visits, I was happy to come because I prefer seeing health messages arrive in the community. (Dr. Rita, female, 54 years old)

Community doctors, such as Dr. Rita, were committed to serving disaster-affected patients displaced in their relatives' houses for several years, helping patients resume their works until they moved to reconstructed sites. However, many tsunami survivors were forced to live in overcrowded displacement camps, which were also often located at a great distance from health centers and clinics. Some patients had previously been displaced due to armed conflict. Therefore, they had been displaced several times, as Dr. Jalal observed.

Most of them were affected by double disasters. Initially, they resided on the mountainside, but they moved to the shore due to armed conflict. But then they were attacked by the tsunami, and they had to return to the mountainside. While living in the shelters, some of them got panic attacks by loud sounds like gunshots. It was a rough time for them. (Dr. Jalal, male, 40 years old)

Displaced patients chose to stay at the camps for different reasons. For example, some patients wanted better access to healthcare and aids, some decided to live closer to their previous settlement, or they did not want to be a 'burden' to their relatives. Living in camps had 
its challenges and advantages. They had to deal with problems, such as overcrowding, a lack of clean water, supplies, and inadequate sanitation facilities [18]. An advantage of living in the camps was access to relatively better healthcare facilities since every camp received visits from mobile clinics. Doctors working in mobile clinics and health centers were required to assist displaced patients in wrestling with the context of the camp.

Community medical doctors working in displacement periods after the tsunami also wrestled with infectious diseases, including malaria. In the following quote, Dr. Anna describes how she employed a community-based approach to providing healthcare for disaster-affected patients with severe malaria infection.

I had several patients that we found unconscious during their displacement periods in a camp far up the mountainside. Previously, their family brought them to a traditional healer to conduct an exorcism to take the 'bad spirit' away from the patients. When it did not work, then the family members contacted me. I visited these patients and took their blood for the rapid test. The result revealed that they had falciparum malaria that had been severely affecting their brain. It was a kind of cerebral malaria. I tried to inject them with Arsucam, a new medicine supplied by an international aid organization. Alhamdulillah, they responded very well. Then I ran a malaria prevention campaign. I was glad that we could distribute mosquito nets; we gave them the 'real things,' so there was no reason to reject the idea anymore. We also explained how to use the Acehnese language to increase people's understanding. (Dr. Anna, female, 50 years old)

During the interview, Dr. Anna mentioned that malaria-associated symptoms were familiar to her. Before the tsunami hit the district, she had encountered many patients with malaria. However, displacement after the tsunami rapidly increased the number of malaria cases due to overcrowding and suboptimal living situations in displacement camps.

Dr. Anna was also familiar with the healthcareseeking behavior of people in her community; they inevitably go to a traditional healer before seeking a medical doctor's help. By that time, the disease may have deteriorated to a severe stage where, in this case, malaria parasites had created inflammation of the brain, causing decreased consciousness, often leading to death. This culture of medical pluralism can have severe consequences for the patient and their family. However, Dr Anna did not blame them for consulting a traditional healer, because she understood that it is their culture, and they had limited choices as their village was located far from healthcare facilities. Therefore, she arranged mobile clinics as soon as possible to provide such a service to improve patient access to better healthcare provision.

The use of an incompatible language in medical encounters was a common problem in communication. Dr. Anna, who originally came from the area, understood the challenges faced by her community. Therefore, she used the local language to promote healthcare and increase people's understanding of malaria prevention measures. With full support from multiple international organizations, Dr. Anna gathered resources to provide disadvantaged people in the community with 'real things' (pre-medicated mosquito nets). Before the tsunami, she could not do this due to limited funds. Like other local doctors in rural areas in Indonesia, Dr. Anna had to wait for the appropriate moment where good intention and facilitation came together. However, she did not use other means of malaria prevention that were aligned with local wisdom, such as stocking ponds and wells with fish that consume mosquito larvae or using particular plants that repel mosquitoes. Her engagement was different from Dr. Julie's, who had more experience with community engagement programs.

In the aftermath of the 2004 Indian Ocean tsunami, Dr. Julie found some survivors from her village displaced in a soccer stadium on higher ground. Initially, her district was located on the shore near the harbor of Banda Aceh. While only approximately 5,000 people in the subdistrict survived the tsunami from 27,000 total occupants, the survivors decided to live together as a community to support each other and improve access to daily logistics at the stadium. Dr. Julie visited them at the stadium from time to time before they moved back to their village several months later. When an international non-government organization from Kuwait reconstructed her health center a year later, Dr. Julie worked there in the district to support the survivors returning to their village. However, the village was not as it was previously because lingering complications of the tsunami overshadowed the culture of the community. Dr. Julie observed how those patients coped with the disaster through a unique reproductive behavior involving herself in many informal conversations with her community members.

I talked with the mothers in informal meetings and understood that the survivors wanted to regain their family by remarrying, and they decided to have children as soon as possible. The birth control program failed [laughing]. There was a local baby boom' period two years after the tsunami. Therefore, instead of conducting a birth control program, our 'mother and child health' team promoted exclusive breastfeeding. We successfully organized a massive event for an exclusive breastfeeding campaign that was attended by 10,000 mothers from all around Aceh from many displacement camps with support from various NGOs. It was a national record! (Dr. Julie, female, 54 years old)

Dr. Julie attempted to learn and understand her community's needs by becoming involved in health programs. Instead of merely conducting centralized health programs assigned by the local health office (birth control program), Dr. Julie adjusted them to the local community's needs. To come to this understanding, she observed the phenomena in the posttsunami community, that is, the urge to remarry and have children. Therefore, she encouraged healthier reproduction behavior by promoting exclusive breastfeeding. The action was an opportunity for better health for babies as the exclusive breastfeeding rate 
before the tsunami was lower than $50 \%$. Dr. Julie and her staff reached out beyond their work area by inviting participants from adjacent districts. After the success of this campaign, Dr. Julie was offered a promotion to work at the local health office. However, she turned it down and continued to work at her community healthcare center.

The above quotes from doctors describe how medical doctors interacted with their patients and disaster-affected community members during the displacement period. Some explained how they came to understand the community's needs by reflecting on their previous experiences. Several doctors applied similar strategies from before the disaster (such as what Dr. Mahmud did), some changed their approaches (similar to Drs. Anna and Julie). Some doctors limited their interventions to an individual level, and others expanded their intervention to the family and community level.

The doctors also tried to apply their cultural competence to increase patient participation. Drs Rita and Anna used the local culture. Dr. Anna attempted to understand medical pluralism cultures and conducted outreach programs to improve the community's engagement with malaria prevention programs. However, her action of informing communities about the importance of mosquito nets is considered a lowlevel of community engagement and may not be wholly capable. Dr. Julie's approach was an example of an engagement encompassing multiple levels, informing patients at personal, family, and community levels. However, despite noble intentions to help patients, every medical interaction has dynamic power relations, especially when the interaction involves marginalized community members, such as disaster-affected patients who are less privileged than ordinary patients. Several cases presented above showed that asymmetrical power relations existed, and further analysis of these cases applying a postcolonial lens may provide valuable insight applicable to improving future medical practice and education.

\section{Discussion}

The findings highlight community engagement's importance in stimulating mutual power-sharing in doctor-patient interactions. The findings complemented what has been described in limited literature of how community engagement works in low-income and developing countries. Building on the research findings, a community engagement model can be developed to inform practitioners working in post-disaster areas in Indonesia and perhaps beyond (Fig. 1.). Deceptively simple, Fig. 1. demonstrates trust-building among community members and other stakeholders, encourages long-term collaboration, and ensures the ownership of healthcare programs belongs to the community rather than NGOs or the government.

Instead of a linear process similar to those produced and used by Health Canada, CTSA, and IAP, as reviewed in the literature review section, this community engagement model utilizes a cyclic, neverending process that emphasizes continuous improvement. Instead of starting from an informed or educated step, it begins with 'listening' to community demands. This step aims to map community vulnerabilities and capacities and locate people's perspectives toward their health problems. It also aims to find indigenous knowledge that has been in place for years as each community's uniqueness.

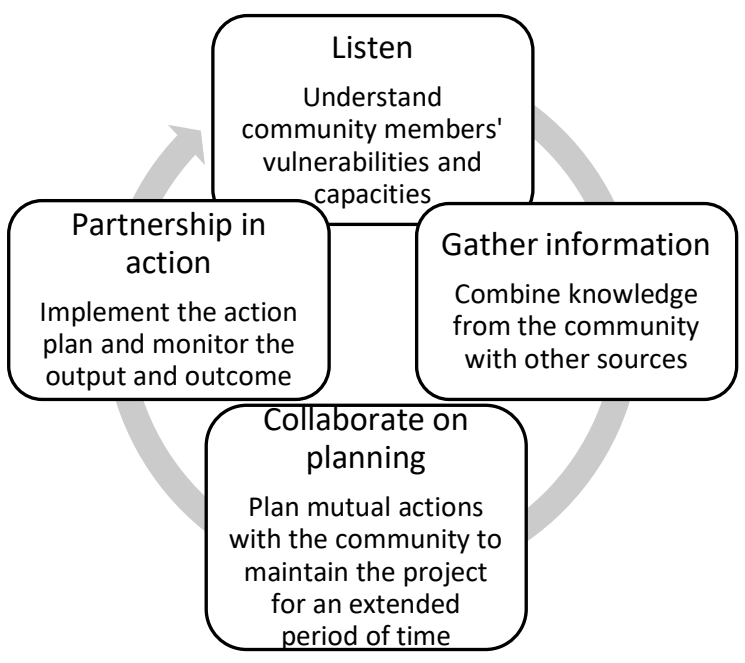

Fig. 2. Proposed model of community engagement suggested by findings of this study.

The community engagement processes work in a cycle. First, listening to the community's needs is essential to identifying health problems and people's perspectives on existing issues and potential solutions. For example, Dr. Julie found the issue of exclusive breastfeeding was more specific and essential to promoting infant health rather than focusing on birth control by carefully listening to the community's demands. Second, health practitioners should gather information on potential accessible resources to resolve health problems, as discussed in the 'listening' step. Dr. Julie gathered information by contacting health stakeholders, including her staff, heads of villages, community representatives, the government, NGOs, and INGOs. Third, collaborating with community members and stakeholders in the planning and executing of health programs. The fourth process is implementing the partnership into action. The partnership requires equal sharing of resources and responsibilities. For instance, Dr. Julie shared duties with neighbors to support older women patients in the community who had mental health issues. This process is reiterated, which involves listening to community responses to the program to improve its implementation.

\section{Conclusion}

Medical doctors are encouraged to continuously pay attention to several aspects of doctor-patient interactions to engage their communities. These aspects include using local languages, participating in cultural activities, and carefully listening to people's voices. These 
approaches may create more balanced power relations, which can help doctors to value each patient's lived experiences as valuable knowledge and an essential resource in healing processes.

The experiences of medical doctors working in postdisaster areas suggest various steps in engaging communities in their health programs. These steps may be described in a cyclic model, comprising listen- gather information-collaborate-partnership- and back to listening to communities' voices to expand the engagement. Instead of linear models established previously, this cyclic model suggests continuing improvement in doctors and community engagement. Based on the experiences of community medical doctor participants, these approaches may help them overcome many challenges in providing integrated post-disaster healthcare services.

This study also calls for medical schools to incorporate community engagement approaches in their curriculum. Hopefully, future doctors will better understand their community needs and improve their willingness and ability to work hand in hand with their communities to overcome more severe challenges in the future.

Acknowledgments. This work was funded by Indonesian Endowment Fund for Education/ Lembaga Pengelola Dana Pendidikan (LPDP) (Grant No. PRJ-1125/LPDP/2015). Dr. Matthew A.M. Thomas and Dr. Ruth Phillips are greatly thanked for inspiring and constructive comments.

\section{References}

1. P. Burns, K. Douglas, W. Hu, P. Aitken, and B. Raphael, "When Disaster Strikes what is the Role of the Local Primary Healthcare Doctor?," Prehospital Disaster Med., vol. 32, no. S1, pp. S70-S71, 2017.

2. S. Abeysinghe, C. Leppold, A. Ozaki, and M. Morita, "Risk, uncertainty and medical practice: changes in the medical professions following disaster," Evid. Policy J. Res. Debate Pract., vol. 16, no. 2, pp. 285-303, 2020.

3. W. Kusumasari, "Technical guidelines for health crisis responses on disaster," in Guidelines for health workers involved in health crisis responses on disaster in Indonesia, The Ministry of Health of Republic of Indonesia Jakarta, 2011, p. 228.

4. J. C. Erves et al., "Needs, priorities, and recommendations for engaging underrepresented populations in clinical research: a community perspective," J. Community Health, vol. 42, no. 3, pp. 472-480, 2017.

5. P. Farmer, Pathologies of power. University of California Press, 2004.

5. A. Ramsbottom, E. O'Brien, L. Ciotti, and J. Takacs, "Enablers and barriers to community engagement in public health emergency preparedness: a literature review," J. Community Health, vol. 43, no. 2, pp. 412-420, 2018.
7. M. D. Morris, S. T. Popper, T. C. Rodwell, S. K. Brodine, and K. C. Brouwer, "Healthcare barriers of refugees post-resettlement," J. Community Health, vol. 34, no. 6, pp. 529-538, 2009.

3. Health Canada, Health Canada Policy Toolkit for Public Involvement in Decision Making. Author Ottawa, ON, Canada, 2000.

7. Clinical and Translational Science Award, "Principles of Community Engagement," Jun. 25, 2015.

https://www.atsdr.cdc.gov/communityengagemen t/pce_ctsa.html (accessed Apr. 29, 2019).

10. International Association for Public Participation, "IAP2 Public Participation Spectrum," 2014. https://www.iap2.org.au/Resources/IAP2Published-Resources (accessed Apr. 29, 2019).

11. E. Manafò, L. Petermann, V. Vandall-Walker, and P. Mason-Lai, "Patient and public engagement in priority setting: a systematic rapid review of the literature," PloS One, vol. 13, no. 3, p. e0193579, 2018.

12. M. Hammersley, "Ethnography: problems and prospects," Ethnogr. Educ., vol. 1, no. 1, pp. 3-14, 2006.

13. R. C. Bogdan and S. K. Biklen, "Research for education: An introduction to theories and methods," 2007.

14. M. D. LeCompte, J. Preissle, and R. Tesch, Ethnography and qualitative design in educational research. Academic Press, 1993.

15. R. Indah, "Insider Dilemmas: An Ethnographic Study on Community-Based Medical Education in Aceh, Indonesia," Explorations, vol. 15, pp. 8692, 2019.

16. R. Indah, "Probing problems: Dilemmas of conducting an ethnographic study in a disasteraffected area," Int. J. Disaster Risk Reduct., vol. 31, pp. 799-805, 2018, doi: https://doi.org/10.1016/j.ijdrr.2018.07.025.

17. H. Arie, B. Syahbudin, and Indonesia. Badan Rehabilitasi \& Rekonstruksi, BRR Book Series: Education, health, women empowerment, preparing quality generation. Executing Agency of Rehabilitation and Reconstruction for Aceh and Nias, 2009. [Online]. Available: https://books.google.co.id/books?id=C5nJxAEA CAAJ

18. E. K. Noji, "Public health issues in disasters," Crit. Care Med., vol. 33, no. 1, pp. S29-S33, 2005. 